\title{
The Effect of Alpha-Lipoic Acid on Diabetic Peripheral Neuropathy and the Upcoming Depressive Disorders of Type II Diabetics
}

Dimitrios T. Karalis ${ }^{1}$, Tilemachos Karalis ${ }^{2}$, Stergios Karalis ${ }^{3}$, Angeliki S. Kleisiari ${ }^{1}$, Foteini Malakoudi ${ }^{3}$, Konstantina Eleni V. Maimari ${ }^{1}$

1. Nutrition and Dietetics, University of Thessaly, Volos, GRC 2. Obstetrics and Gynecology, General Hospital of Trikala, Trikala, GRC 3. Internal Medicine, General Hospital of Trikala, Trikala, GRC

Corresponding author: Dimitrios T. Karalis, karalis_dim@yahoo.gr

\section{Abstract}

\section{Introduction}

Peripheral neuropathy is one of the possible complications of diabetes. Alpha-lipoic acid (a-lipoic acid or ALA) is a powerful antioxidant cofactor synthesized in mitochondria that could help stimulate nerves and regenerate nerve fibers, thus preventing disease progression. Moreover, the possible feeling of oppression from the lifestyle changes needed to avoid the complications of diabetes may contribute to the development of depressive symptoms. ALA increases insulin sensitivity, which could increase serotonin synthesis and thus reduce the manifestations of depressive disorder.

\section{Aim}

The aim of this study is to investigate the therapeutic effect after oral administration of a-lipoic acid in patients with type II diabetes mellitus, regarding the possibility of developing peripheral neuropathy and the possibility of developing depressive disorder due to the existence of diabetes type II.

\section{Methods}

The study sample consisted of 148 Greek patients, type II diabetics, 68 men and 80 women, aged 50-75 years. All of them were non-smokers and did not consume alcohol. Their treatment was a combination of gliclazide, sodium-glucose-linked transporter 2 (SGLT-2) inhibitors, metformin, and glucagon-like peptide 1 (GLP-1) analogs. None of them were under insulin administration. Any other treatment received chronically from the patients for other comorbidities was not altered or paused. All patients were in regular monitoring of renal, hepatic, and ocular function, which was normal. Patients were monitored with a balanced diet, based on equivalents, in order to maintain an almost constant body mass index (BMI). All were given one tablet of $600 \mathrm{mg}$ a-lipoic acid, two hours before a meal, for eight months, and the incidence of developing peripheral neuropathy and depressive disorder was assessed, using the Subjective Peripheral Neuropathy Screen Questionnaire (SPNSQ) and Beck Depression Inventory (BDI) questionnaire.

Review began $11 / 18 / 2020$ Review ended 01/12/2021 Published 01/18/2021

๑) Copyright 2021

Karalis et al. This is an open access article distributed under the terms of the Creative Commons Attribution License CC-BY 4.0., which permits unrestricted use, distribution, and reproduction in any medium, provided the original author and source are credited.

\section{Results}

ALA administration after both four and eight months resulted in statistically significant results and, specifically, the peripheral neuropathy development mean score was reduced by 4.79 at four months and 6.22 after eight months. Concerning the incidence of depressive disorder, an average decrease of 4.43 in the related depression score was observed at the four-month milestone and 7.56 at eight months, both statistically significant.

\section{Conclusion}

A-lipoic acid is a powerful antioxidant and, when used with conventional treatment, has shown to significantly decrease the incidence of depression and peripheral neuropathy in patients with type 2 diabetes mellitus.

Categories: Endocrinology/Diabetes/Metabolism, Neurology, Psychiatry

Keywords: alpha lipoic acid, diabetes type 2, type ii diabetes, diabetic complications, diabetic neuropathy, depression

\section{Introduction}

Alpha-lipoic acid (a-lipoic acid or ALA) is a cofactor found under normal conditions in most eukaryotic and prokaryotic organisms. It is synthesized in the mitochondria. It is essential for the proper functioning of several enzymes that play a key role in oxidative metabolism [1-2]. In particular, it is a powerful antioxidant that is soluble in both water and fat; thus, it is easily absorbed after oral administration [3-6]. 
It was first isolated in 1950 by Reed and colleagues from bovine liver as a catalyst associated with pyruvate dehydrogenase [7]. In 1992, Reed and Cronan for the first time identified the DNA sequence of two genes, lipA and lipB, which were involved in the biosynthesis and metabolism of ALA as a part of the Escherichia coli genome [8]. Subsequent analyses of the Escherichia coli genome developed further knowledge of the pathways of the synthesis, adhesion, and functioning of ALA [9].

The human body cannot synthesize sufficient amounts of ALA to meet its needs. Thus, it is necessary to obtain it from dietary sources [10]. The bioavailability of ALA decreases significantly with food intake. It is, therefore, recommended to administer it at least two hours before eating; if this is not possible, at least 30 minutes post-meal [11]. This is mainly because ALA, being a weak acid, needs the acidic $\mathrm{pH}$ of the stomach to be absorbed readily. Food interferes and competes with the absorption [11-13].

One of the complications of diabetes is peripheral neuropathy. It is an aesthetic-motor polyneuropathy with progressive severity of symptoms that results in functional decline and reduced quality of life [14-15]. As a powerful antioxidant factor, ALA helps stimulate nerves and regenerate nerve fibers, thus preventing the progression of diabetic neuropathy and helping alleviate its symptoms [16-17].

Depression has been related to altered levels of neurotransmitters in the human brain, in particular, serotonin [18-20]. Serotonin and other monoamine neurotransmitters are synthesized from the amino acid precursor tryptophan [21]. Insulin as a whole increases the influx of tryptophan in the brain, resulting in the synthesis of serotonin [22]. In diabetic patients, the reduced secretion or reduced activity of insulin relates to the reduction of the ratio of tryptophan to other plasma amino acids, resulting in a reduced availability of tryptophan in the brain and, therefore, a decrease in serotonin concentration [23]. Thus, increasing insulin sensitivity may lead to increased tryptophan availability and, therefore, an increase in the production of serotonin and other monoamines in the brain [22]. Due to its ability to increase insulin sensitivity, ALA could help improve the influx of tryptophan into the brain, causing an increase in serotonin synthesis and resulting in a reduction in depression [24].

In the present study, we will try to investigate the therapeutic effect of oral a-lipoic acid in patients with type II diabetes, in terms of the incidence of peripheral neuropathy and depressive disorder.

\section{Materials And Methods}

The study sample consisted of 148 patients, 68 men and 80 women in the age group of 50-75 years. The study included patients in the healthy weight range (body mass index (BMI) $18-25 \mathrm{~kg} / \mathrm{m}^{2}$ ), overweight (BMI $25-29.9 \mathrm{~kg} / \mathrm{m}^{2}$ ), and obese patients (BMI $>30 \mathrm{~kg} / \mathrm{m}^{2}$ ). All subjects in the study declared that they were non-smokers and did not consume alcohol. Inclusion criteria in the study were the existence of type II diabetes (based on the diagnostic criteria set by the World Health Organization), not taking insulin for glycemic control, and the absence of mental disabilities in order to achieve a correct subjective assessment of the complications of diabetes mellitus. All patients received $600 \mathrm{mg}$ of a-lipoic acid daily, in tablet form, instructed to take it at least two hours before meals, for a period of eight months, and the possibility of peripheral neuropathy and depressive disorder was evaluated. The diabetic management of each patient remained the same as before the study. Patients were on a combination of various drugs, including gliclazide (sulfonylurea), sodium-glucose linked transporter 2 (SGLT-2) inhibitors, metformin, and glucagon-like peptide 1 (GLP-1) analogs. Patients on antihypertensive and lipid-lowering drugs continued their treatments. In addition, all volunteers had good renal and hepatic function and were under medical supervision for the early diagnosis and treatment of possible health problems. Finally, all participants were informed about the aims of the study, as well as about the process of measurements, evaluations using questionnaires, and the medical examinations to which they would be submitted and gave their approval for their participation, through written consent (according to the protocols of the Research Ethics and Conduct Committee).

The height of the participants was measured, once, using a built-in stature meter in an upright position, without shoes, and with an accuracy of $0.5 \mathrm{~cm}$. Weight was measured in light clothing and without shoes, on an electronic precision balance, and the measurements were rounded to the nearest hundred grams. The scales were adjusted and checked before and after each weighing. The body mass index (BMI) was then calculated as the quotient of weight (measured in kilograms, $\mathrm{kg}$ ) to the square of height (measured in meters, $\mathrm{m})$.

Two questionnaires were selected as the most appropriate tools for the evaluation of possible peripheral neuropathy and depression. The Subjective Peripheral Neuropathy Screen Questionnaire (SPNSQ) was selected to assess the likelihood of developing peripheral neuropathy, which consists of 15 "yes/no" questions about the symptoms of the neuropathy, and the score is calculated by summation of the "yes" answers. The score ranges from " 0 " (no neuropathic symptoms) to "15" (the most severe neuropathic symptoms). The "Aaron T. Beck questionnaire" (Beck Depression Inventory - BDI), which is a selfassessment questionnaire consisting of 21 multiple-choice questions, was selected to assess the presence of depression. It is a widely used instrument for measuring the severity of depression. Each response is scored on a scale from 0 to 3 . Higher overall scores indicate more severe depressive symptoms. The overall score is 


\section{Cureus}

evaluated as follows: 0-13: minimal depression, 14-19: mild depression, 20-28: moderate depression, and 29-63: severe depression.

The diet of each patient was monitored. They were given a balanced diet in order to maintain a relatively stable body mass index during the eight months of the study. In addition, they were given general dietary instructions based on the special needs of the diabetic population.

The analysis of the research results was done with the Statistical Package for the Social Sciences (SPSS) (Version 25, IBM Corporation, Armonk, NY). The research sample consisted of 148 patients $(\mathrm{N}=148)$. For the correlation test between the two periods, from zero to four months and from zero to eight months, Pearson's linear coefficient was applied to the data with values being significant at $<0.05$ (correlation positive at $\mathrm{p}<0.05)$. Results were evaluated in the form of tables and graphs.

\section{Results}

A statistically significant decrease in the score points of the BDI questionnaire was observed in both time periods. For the first period (four months after the administration of ALA), an average decrease of 4.43 points was recorded (mean reduction $=4.43$, percentage $22.7 \%$ ). The results were statistically significant $(\mathrm{p}=$ $0.00001<0.05$ ). For the second period (eight months after the administration of ALA), an average decrease of 7.56 points was recorded as compared to day 0 (mean reduction $=7.56$, percentage $38.7 \%$ ). The results were also statistically significant $(\mathrm{p}=0.00001<0.05)$. The results are shown in Table 1 and Figure 1 .

\begin{tabular}{|c|c|c|c|c|c|}
\hline & $\mathbf{N}$ & Minimum Value & Maximum Value & Mean Value & Std. Deviation \\
\hline BDI before & 148 & 5 & 35 & 19.51 & 5,920 \\
\hline BDI 4 months later & 148 & 2 & 32 & 15.08 & 6,214 \\
\hline BDI 8 months later & 148 & 1 & 33 & 11.95 & 6,384 \\
\hline Valid N (listwise) & 148 & & & & \\
\hline
\end{tabular}

TABLE 1: Descriptive statistical measures for BDI questionnaire score (zero, four, and eight months)

BDI: Beck Depression Inventory

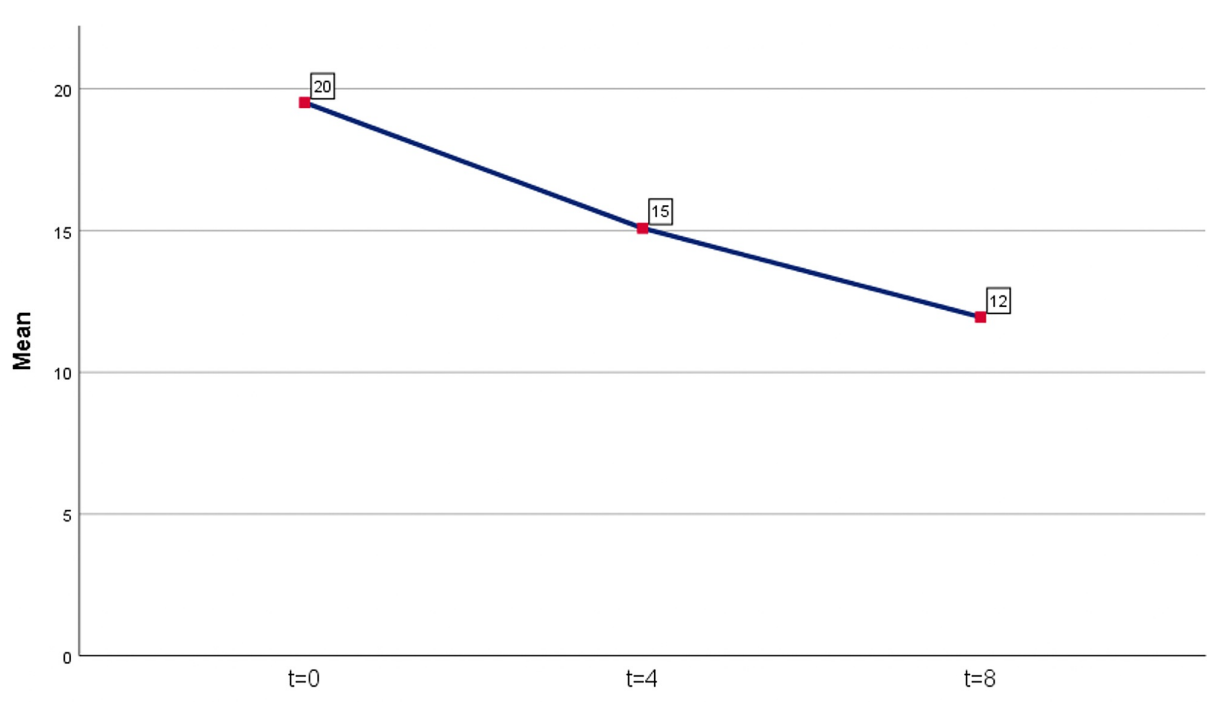

FIGURE 1: Average BDI levels (rounded, no decimals)

BDI: Beck Depression Inventory

A statistically significant decrease in the score points of the SPNSQ questionnaire was observed in both time periods. For the first period (four months after the administration of ALA), an average decrease of 4.79 points was recorded (mean reduction $=4.79$, percentage $63.7 \%$ ). The results were statistically significant $(p=$ 


\section{Cureus}

$0.00001<0.05$ ). For the second period (eight months after the administration of ALA), an average decrease of 6.22 points as compared to day 0 was recorded (mean reduction $=6.22$, percentage $82.7 \%$ ). The results were also statistically significant $(\mathrm{p}=0.00001<0.05)$. The results are shown in Table 2 and Figure 2 .

\begin{tabular}{|c|c|c|c|c|c|}
\hline & $\mathbf{N}$ & Minimum Value & Maximum Value & Mean Value & Std. Deviation \\
\hline SPNSQ before & 148 & 3 & 15 & 7.52 & 2,737 \\
\hline SPNSQ 4 months later & 148 & 0 & 12 & 2.73 & 2,032 \\
\hline SPNSQ 8 months later & 148 & 0 & 6 & 1.30 & 1,249 \\
\hline Valid N (listwise) & 148 & & & & \\
\hline
\end{tabular}

\section{TABLE 2: Descriptive statistical measures for the SPNSQ score (zero, four, and eight months)}

SPNSQ: Subjective Peripheral Neuropathy Screen Questionnaire

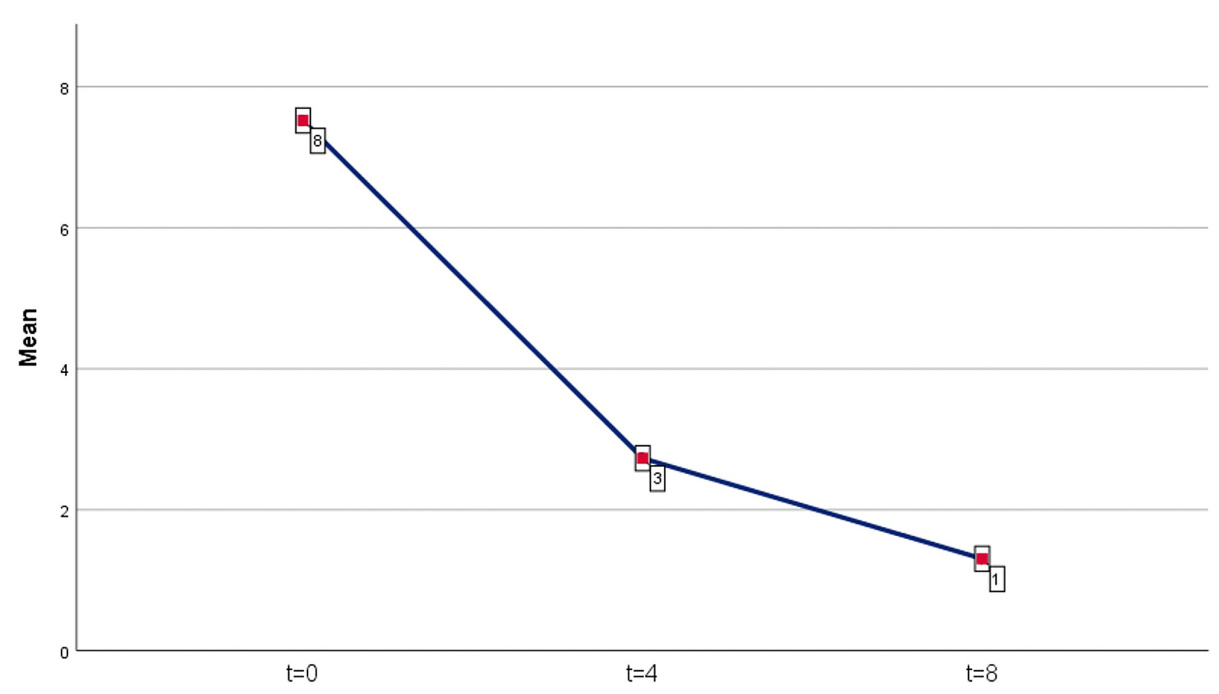

FIGURE 2: SPNSQ level average (rounded, no decimals)

SPNSQ: Subjective Peripheral Neuropathy Screen Questionnaire

\section{Discussion}

This study investigated the therapeutic effect of orally administered $600 \mathrm{mg}$ a-lipoic acid on depression and peripheral neuropathy related to diabetes. It concluded that there is a statistically significant improvement in the prevalence of depression and peripheral neuropathy with the administration of $600 \mathrm{mg}$ a-lipoic acid daily in diabetics.

The positive effect of a-lipoic acid has been well-researched and proven in peripheral neuropathy. A study conducted in 2002 evaluating the safety and efficacy of a-lipoic acid in the total symptom score (TSS), a measure of positive neuropathic sensory symptoms, demonstrated an improvement of the TSS score of the ALA receiving group from the baseline by an average of 5.7 points as compared to the group receiving placebo that had an average of 1.8 points [25]. Another study that evaluated the progression of diabetic neuropathy after the long-term administration of ALA during a period of four years demonstrated that participants taking ALA showed stabilization of the disease, in contrast with the conventional therapies for the treatment of symptoms, such as duloxetine, pregabalin, and gabapentin, which did not appear to stop the progression of the condition [26]. Finally, a meta-analysis of four double-blind placebo-controlled studies involving more than 1200 patients showed that a-lipoic acid at a dose of $600 \mathrm{mg}$ daily significantly improved the clinical picture of patients with diabetic neuropathy [27].

We found significant improvement in the depressive symptoms of our patients, which is a finding supported by previous literature. A review study evaluating the potential of ALA administration in various psychiatric and neurological diseases and symptoms concluded that ALA is a promising adjuvant for treating 
depression as demonstrated by the hormonal fluctuations in the prefrontal cortex of mice models [28]. Finally, a study of mice models with pharmaceutically induced depression demonstrated that the oral administration of ALA through a feeding tube increased the levels of brain-derived neurotrophic factor (BDNF), similar to anti-depressants, supporting that ALA administration could have an anti-depression potential [29].

\section{Conclusions}

In conclusion, the present study supports the existing research claims for the significant benefits of a-lipoic acid administration in reducing the likelihood of developing peripheral neuropathy in diabetic patients. In addition, the results of the study show that the daily administration of a-lipoic acid results in a significant reduction in depressive symptoms. Further clinical trials are needed to demonstrate whether the benefits of a-lipoic acid supplementation can be long term in diabetic populations as well as in individuals with diseases strongly associated with the presence of oxidative stress in the human body.

\section{Additional Information \\ Disclosures}

Human subjects: Consent was obtained or waived by all participants in this study. National Ethics Committee for Clinical Studies issued approval 55480/6-09-2006. The Greek legislation concerning interventional clinical trials on investigational medicinal products (CTIMPs) was published in the Greek Republic Gazette No 1973 of 31 December 2003. It refers to the Minister of Health's decision DYG 3/89292, which describes the law under which the Directive 2001/20/EC was adopted in Greece. The law has no title, as all laws in Greece are referred to by number. Standard Operating Procedures for the National Ethics Committee were published in the Greek Republic Gazette No 1503 of 7 October 2004, referring to the Minister of Health's decision DYG 3(A) 69150. Issued protocol number No 1503. Animal subjects: All authors have confirmed that this study did not involve animal subjects or tissue. Conflicts of interest: In compliance with the ICMJE uniform disclosure form, all authors declare the following: Payment/services info: All authors have declared that no financial support was received from any organization for the submitted work. Financial relationships: All authors have declared that they have no financial relationships at present or within the previous three years with any organizations that might have an interest in the submitted work. Other relationships: All authors have declared that there are no other relationships or activities that could appear to have influenced the submitted work.

\section{References}

1. Reed LJ, Hackert ML: Structure-function relationships in dihydrolipoamide acyltransferases . J Biol Chem. 1990, 265:8971-8974.

2. Morris TW, Reed KE, Cronan JE Jr: Lipoic acid metabolism in Escherichia coli: the lplA and lipB genes define redundant pathways for ligation of lipoyl groups to apoprotein. J Bacteriol. 1995, 177:1-10. 10.1128/jb.177.1.1-10.1995

3. Packer L, Witt EH, Tritschler HJ: Alpha-Lipoic acid as a biological antioxidant . Free Radic Biol Med. 1995, 19:227-250. 10.1016/0891-5849(95)00017-r

4. Packer L, Tritschler HJ: Alpha-lipoic acid: the metabolic antioxidant. Free Radic Biol Med. 1996, 20:625-626. 10.1016/0891-5849(95)02129-9

5. Müller L, Menzel H: Studies on the efficacy of lipoate and dihydrolipoate in the alteration of cadmium2 + toxicity in isolated hepatocytes. Biochim Biophys Acta. 1990, 1052:386-391. 10.1016/0167-4889(90)90147-6

6. Islam M: Antioxidant activities of dithiol alpha-lipoic acid . Bangladesh J Med Sci. 2009, 8:46-51. 10.3329/bims.v8i3.3982

7. Reed LJ, DeBusk BG, Gansalus IC, Hornberger Cs Jr: Crystalline alpha-lipoic acid; a catalytic agent associated with pyruvate dehydrogenase. Science. 1951, 114:93-94. 10.1126/science.114.2952.93

8. Vanden Boom TJ, Reed KE, Cronan JE Jr: Lipoic acid metabolism in Escherichia coli: isolation of null mutants defective in lipoic acid biosynthesis, molecular cloning and characterization of the E. coli lip locus, and identification of the lipoylated protein of the glycine cleavage system. J Bacteriol. 1991, 173:6411-6420. 10.1128/jb.173.20.6411-6420.1991

9. Cronan JE: Biotin and lipoic acid: synthesis, attachment, and regulation . EcoSal Plus. 2008, 3:[Epub]. 10.1128/ecosalplus.3.6.3.5

10. Shay KP, Moreau RF, Smith EJ, Smith AR, Hagen TM: Alpha-lipoic acid as a dietary supplement: molecular mechanisms and therapeutic potential. Biochim Biophys Acta. 2009, 1790:1149-1160. 10.1016/j.bbagen.2009.07.026

11. Gleiter CH, Schug BS, Hermann R, Elze M, Blume HH, Gundert-Remy U: Influence of food intake on the bioavailability of thioctic acid enantiomers. Eur J Clin Pharmacol. 1996, 50:513-514. 10.1007/s002280050151

12. Singh U, Jialal I: Alpha-lipoic acid supplementation and diabetes. Nutr Rev. 2008, 66:646-657. 10.1111/j.1753-4887.2008.00118.x

13. Brufani M: $\alpha$-lipoic acid: drug or dietary supplement? An overview on the pharmacokinetics, available formulations and clinical evidence in the diabetes complications. Progr Nutr. 2014, 16:62-64.

14. Dyck PJ, Kratz KM, Karnes JL, et al.: The prevalence by staged severity of various types of diabetic neuropathy, retinopathy, and nephropathy in a population-based cohort: the Rochester Diabetic Neuropathy Study. Neurology. 1993, 43:817-824. 10.1212/wnl.43.4.817

15. Abbott CA, Malik RA, Ernest RE, Kulkarni J, Boulton AJM: Prevalence and characteristics of painful diabetic neuropathy in a large community-based diabetic population in the UK. Diabetes Care. 2011, 34:2220-2224. 


\section{Cureus}

10.2337/dc11-1108

16. Nishikawa T, Edelstein D, Du XL, et al.: Normalizing mitochondrial superoxide production blocks three pathways of hyperglycaemic damage. Nature. 2000, 404:787-790. 10.1038/35008121

17. Murase K, Hattori A, Kohno M, Hayashi K: Stimulation of nerve growth factor synthesis / secretion in mouse astroglial cells by coenzymes. Biochem Mol Biol Int. 1993, 30:615-621.

18. Coppen A: The biochemistry of affective disorders. Br J Psychiatry. 1967, 113:1237-1264. 10.1192/bjp.113.504.1237

19. Roy A, DeJong J, Linniola M: Cerebrospinal fluid monoamine metabolites and suicidal behavior in depressed patients. Arch Gen Psychiatry. 1989, 46:609-612. 10.1001/archpsyc.1989.01810070035005

20. Kerr CW: The serotonin theory of depression. Jefferson Journal of Psychiatry. 1994, 12:4. 10.29046/JJP.012.1.001

21. Carlsson A, Lindqvist M: The effect of L-tryptophan and some psychotropic drugs on the formation of 5hydroxytryptophan in the mouse brain in vivo. J Neural Transm. 1972, 33:23-43. 10.1007/BF01244726

22. Chaouloff F: The serotonin hypothesis. Series in health psychology and behavioral medicine. Physical activity and mental health. Morgan, WP (ed): Taylor and Francis, Washington, DC; 1997. 179-198.

23. Kern, S, Engerman RL: Abnormal amino acid concentrations in plasma and urine of experimentally diabetic dogs. Research in Experimental Medicine. 1983, 182:185-192. 10.1007/BF01851707

24. Salazar MR: Alpha lipoic acid: a novel treatment for depression . Med Hypotheses. 2000, 55:510-512. 10.1054/mehy.2000.1103

25. Alexander SA, Alexei B, Peter JD, et al.: The sensory symptoms of diabetic polyneuropathy are improved with $\alpha$-lipoic acid. Diabetes Care. 2003, 26:770-776. 10.2337/diacare.26.3.770

26. Ziegler D, Low PA, Litchy WJ, et al.: Efficacy and safety of antioxidant treatment with $\alpha$-lipoic acid over 4 years in diabetic polyneuropathy. The NATHAN 1 trial. Diabetes Care. 2011, 34:2054-2060. 10.2337/dc110503

27. Ziegler D, Nowak H, Kempler P, Vargha P, Low PA: Treatment of symptomatic diabetic polyneuropathy with the antioxidant alpha-lipoic acid: a meta-analysis. Diabet Med. 2004, 21:114-121. 10.1111/j.14645491.2004.01109.x

28. Cekici H: Potential therapeutic agent in psychiatric and neurological diseases: alpha lipoic acid . Acta Psychopathol. 2018, 4:9. 10.4172/2469-6676.100165

29. Ramalho FMHN, De Sousa CNS, Meneses LN, et al.: P.1.g.082. Antidepressant effect of alpha-lipoic acid: brain-derived neurotrophic factor as a new target for resistant depression. Eur Neuropsychopharmacology. 2014, 24:251. 10.1016/S0924-977X(14)70393-1 